\title{
High-fidelity Modelling of Self-regulating Pneumatic Valves
}

\author{
Alexander Pollok ${ }^{1}$ Francesco Casella ${ }^{2}$ \\ ${ }^{1}$ Institute of System Dynamics and Control, German Aerospace Center (DLR), Germany, \\ alexander.pollok@dlr.de \\ ${ }^{2}$ Dipartimento di Elettronica, Informazione e Bioingegneria, Politecnico di Milano, Italy, \\ francesco.casella@polimi.it
}

\begin{abstract}
In conventional aircraft energy systems, self-regulating pneumatic valves (SRPVs) are used to control the pressure and mass flow of the bleed air. The dynamic behavior of these valves is complex and dependent on several physical phenomena. In some cases, limit cycles can occur, deteriorating performance. This paper presents a complex multiphysical model of SRPVs implemented in Modelica. First, the working-principle is explained, and common challenges in control-system design-problems related to these valves are illustrated. Then, a Modelicamodel is presented in detail, taking into account several physical domains. It is shown, how limit cycle oscillations occurring in aircraft energy systems can be represented with this model. Finally, some multi-domain interactive effects are described.

Keywords: Modelica, Thermofluid, Modeling, Friction, Electrohydraulic, Hydraulic
\end{abstract}

\section{Introduction}

In applications related to process control often relatively simple valve models are used. They are based on flow coefficients, and relate mass flow to pressure drop by the use of a quadratic relationship. This helps keeping the system model at a low-order, benefitting understanding as well as control design. Most of the time, these simple models are accurate enough, and all relevant dynamics are included.

There are however applications, where simple models are inadequate. This can be the case, if high accuracy is needed, when choking occurs, or when internal valve phenomena are relevant. Neglection of these cases, and the utilization of an inadequate model can lead to unwanted behavior in the controlled system: Valve dynamics often contain nonlinearities like stiction, backlash and deadband, which in turn can lead to oscillations (Choudhury et al., 2006).

Indeed, according to Bialowski (1993), about 30\% of controlled loops in the process industry are oscillating. In Desborough and Miller (2002), 26.000 PID con- trollers in the process industry are surveyed: $16 \%$ are classified as excellent, $16 \%$ as acceptable, $22 \%$ as fair, $10 \%$ as poor, and $36 \%$ run in open-loop.

In aircraft, SRPVs are used to control the pressure and flow rate of the engine bleed air. An illustration of the working principle can be found in Figure 1, more detailed descriptions can be found in Section 2.

SRPVs operate under harsh conditions inside the engine nacelle. Since several SRPVs are operated in-line, their dynamic behavior has to be tuned so as to avoid the occurrence of limit cycles. This can be done in situ, but the associated costs are substantial. Being able to predict the system behavior better during the design phase would reduce those costs considerably, but for a sufficient level of prediction-accuracy a high-fidelity model is needed.

Related research has been done by several authors. Beater (2000) presented a simple model of an electrohydraulic valve in Modelica and HyLib. In Beater and Clauß (2003), a pneumatic drive system is modelled in Modelica, combining pneumatic, mechanical and electronic domains. A free-piston-engine modelled in Modelica is described in Pohl and Gräf (2005), containing detailed submodels of several physical domains. PujanaArrese et al. (2007) presented a Modelica-model of a pneumatic muscle, combining fluid modelling with the mechanical system of kinematics.

The goal of this paper is to demonstrate how highfidelity multi-physical models of self-regulating pneumatic valves can be developed in the object-oriented equation-based modelling-language Modelica. It is structured as follows: In Section 2, the Modelica model for SRPVs is presented and the motivations for modelling choices are explained, subdivided into the different physical domains. Libraries, models and implementations that are used in this work are mentioned. In Section 3 , exemplary model outputs are shown, and a number of emerging phenomena are discussed. The paper is concluded in Section 4. 


\section{Valve Modelling}

\subsection{Functioning Principle}

The main functioning principle of a self-regulating pneumatic valve is based on automatic pressure balancing. A small pipe connects the main pipe downstream of the valve with the lower end of the valve actuator chamber. Inside the chamber, a piston divides the chamber into two volumes.

The piston is connected to the butterfly valve disk by a mechanical mechanism. In this way, if the downstream pressure increases, the pressure in the lower part of the chamber increases as well and moves the piston upwards. This closes the valve disk, leading to a lower valve mass flow. Depending on the flow configuration outside of the valve scope, this usually decreases the downstream pressure, closing the pneumatic control loop.

Additionally, a second control loop is present. By the use of pressure-reducers, vents, and/or small electric regulating valves, air can flow from upstream of the valve to the upper chamber, or from the upper chamber to the ambient. The implementation of the second loop can differ by a great deal, in this work two implementations are modelled:

1. pneumatic actuator: a pneumatic system using two pressure-reducers keeps the pressure in the upper chamber inside a predefined interval

2. electro-pneumatic actuator: a PID-controller directly imposes the air mass-flow from or into the upper chamber

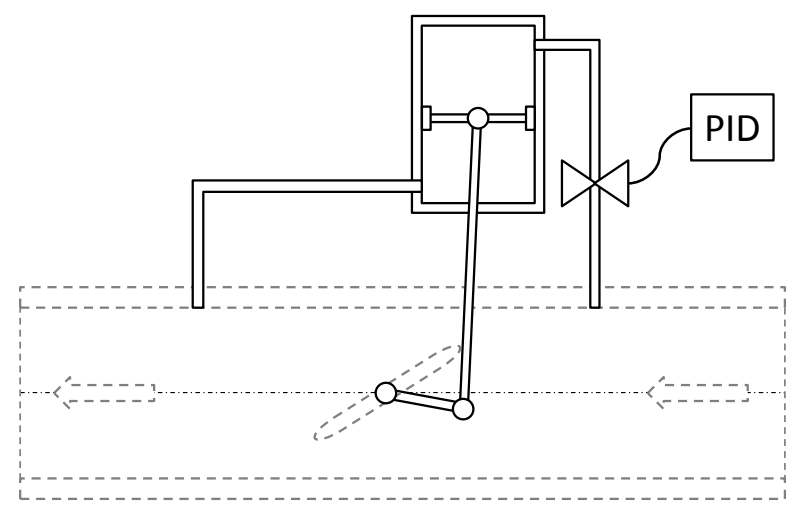

Figure 1. Illustration of a self-regulating pneumatic valve

For the sake of clarity, the top-level model of the selfregulating pneumatic valve has been split into two parts: one valve-part and one actuator-part. The partitioning is illustrated in Figure 1, where the valve part is depicted in dashed-grey lines.

\subsection{Detailed Valve model}

The valve model calculates the mass flow through the valve depending on the up- and downstream fluid properties and the valve angle. The symbol and the connectors of the valve model are depicted in Figure 2.

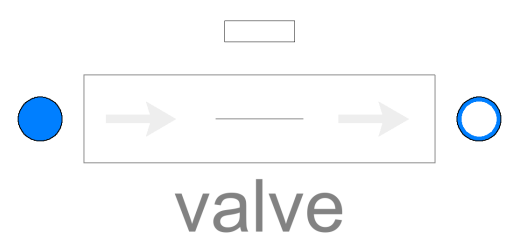

Figure 2. Modelica symbol layer of the valve model

In aircraft bleed air systems, flow velocity is quite high. Physical effects of high-speed compressible flow cannot be neglected (Sielemann, 2012a), so the capabilities of the Modelica Fluid library are not sufficient. Thus, as fluid interface, higher-order stencil-based connectors for gas-dynamics as presented in Sielemann (2012b) are used. These connectors include far more information than the connectors from the Modelica Fluid library: For a variable number of fluid cells, pressure, temperature and fluid velocity are included. This is illustrated in Figure 3. With this information, higher order discretization schemes of computational fluid dynamics can be used.

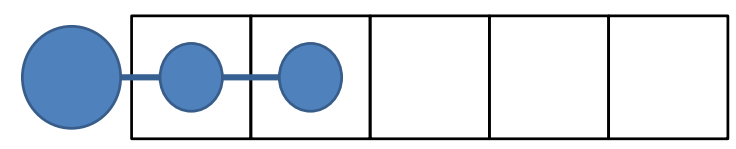

Figure 3. Illustration of the gas-dynamics connector principle, including information about multiple volume cells

For the mass flow calculation potentially choked flow effects have to be taken into account. Therefore the standard calculation using flow coefficients is discarded. Instead, a flow function approach is used, based on an enthalpy-balance and adiabatic state change. The corresponding Modelica function can be seen in Listing 1 . The result of this function is multiplied with a factor that is dependent on the valve angle.

Listing 1. Modelica code of the valve mass flow calculation

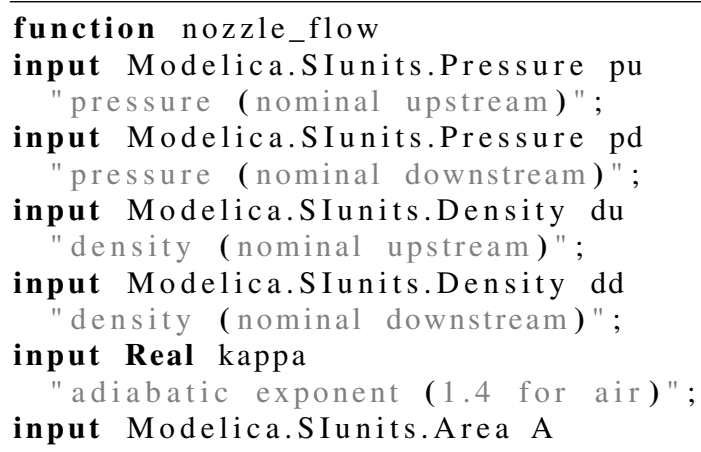




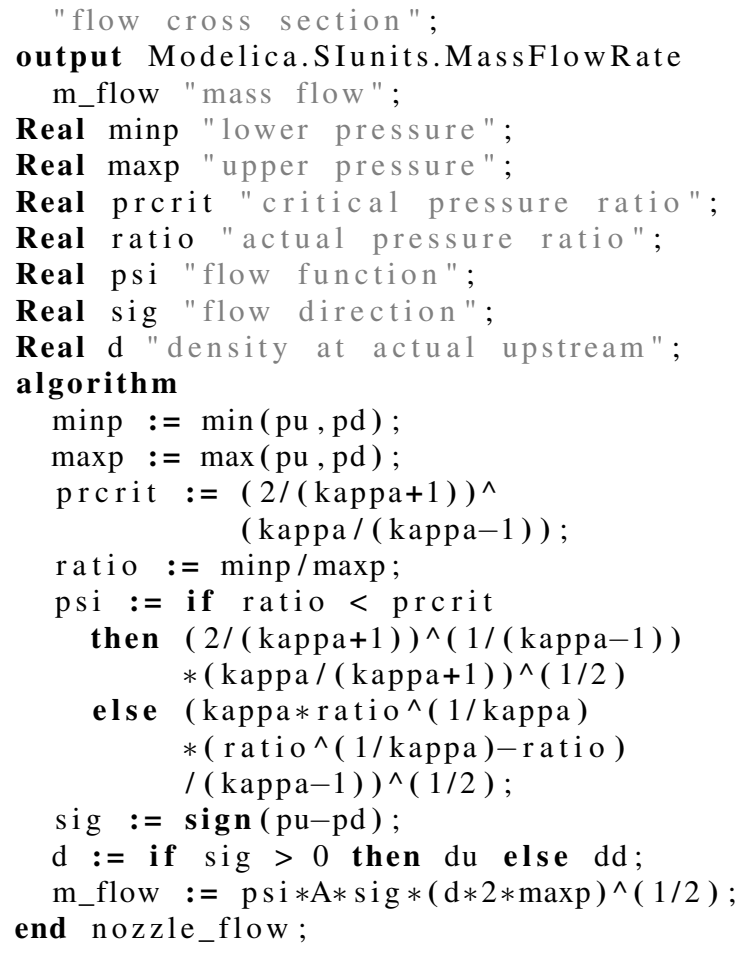

Fluids moving through a butterfly valve at high velocities induce a fluiddynamic torque on the valve disk. This generates an interesting coupling between the fluid and mechanic domains of a valve model. For the calculation of the torque, two approaches are often used: one based on the pressure difference, one based on the fluid velocity. In Solliec and Danbon (1999), the different approaches are compared. We use the classical approach based on pressure difference, as the pressure difference is more clearly defined than the fluid velocity in the context of lumped parameter models. Here, the torque $\mathrm{T}$ is calculated as:

$$
T(\alpha)=K(\alpha) \cdot \Delta P \cdot D^{3}
$$

where $\mathrm{K}$ is the torque coefficient, $\Delta P$ is the pressure difference, $\alpha$ is the valve angle and $\mathrm{D}$ is the valve diameter. A spline-based approach is used to describe the dependency between torque coefficient and valve angle. A Modelica multibody connector provides the valve angle and feeds back the induced fluiddynamic torque.

\subsection{Actuator model}

Two actuator models as described in Section 2.1 are needed, for two different implementations of the second control loop. Accordingly, one partial model together with two extending models was created. The Modelica diagram of the base model can be seen in Figure 4.

Three physical domains are significant for the modelling of the valve actuator: the fluid dynamics inside the chambers, the multi-body mechanics of the mechanism, and the thermal behavior of the parts. They are connected through the piston and chamber components,

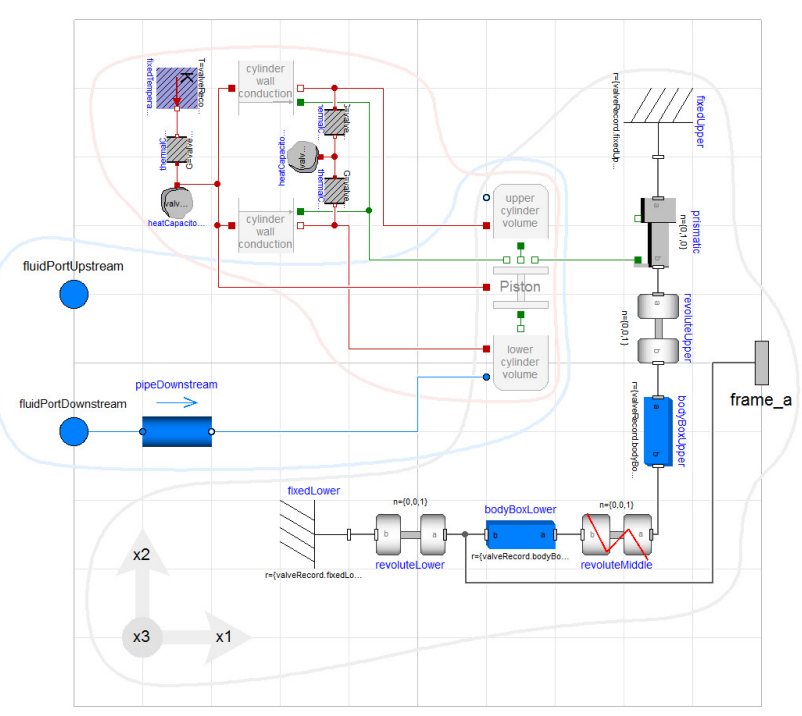

Figure 4. Modelica component layer of the (partial) valve actuator base model

where all domains have considerable influence. The domains are indicated in Figure 4 through colored lines.

\subsubsection{Mechanical domain}

The core of the mechanical domain is the piston-model, where a one-dimensional force balance over the piston is calculated, see Equation 2. The occurring forces are commented in the following:

$$
\begin{aligned}
& F_{\text {pressure }_{\text {upper }}}+F_{\text {pressure }_{\text {lower }}}+F_{\text {constraint }} \\
& +F_{\text {friction }}+F_{d^{\prime} \text { alembert }}+F_{\text {joint }}=0
\end{aligned}
$$

Pressure forces:

The piston model and both chamber models are connected by translational mechanical connectors. In this way, the position and the forces generated by fluid pressure are exchanged.

Constraining forces:

Based on the construction, the movement allowance of the piston is limited. To represent this, stiff quadratic spring forces are implemented. These come into effect as soon as the end of the stroke is reached.

Friction force:

The friction forces between piston and cylinder are mainly responsible for unwanted stiction-effects. Detailed modelling of friction phenomena is therefore necessary. Furthermore, a simple model based on two static and dynamic friction coefficients is numerically unfavourable when the piston position is used as a state. In this work, we used the LundGrenoble (Lu-Gre) friction model (De Wit et al., 
1995). It is a detailed model of friction with internal states that represent the deflection of the bristles (micro-bumps in the material surface). The implementation in Modelica was done according to Aberger and Otter (2002), but instead of rotatory coordinates, translative coordinates were used. An example trajectory of friction force over piston velocity can be seen in Figure 5.

d'Alembert force:

The d'Alembert force, or inertial force, of the piston is calculated by deriving the position w.r.t. time two times and multiplying with its mass. Of course, this makes the system quite stiff from a numerical point of view, but then, there are solvers of production-quality available to handle stiff systems.

Joint force:

The joint force is the linking force between the translative piston dynamics and the planar dynamics of the mechanism. The prismatic joint model of the multibody library provides the interface.

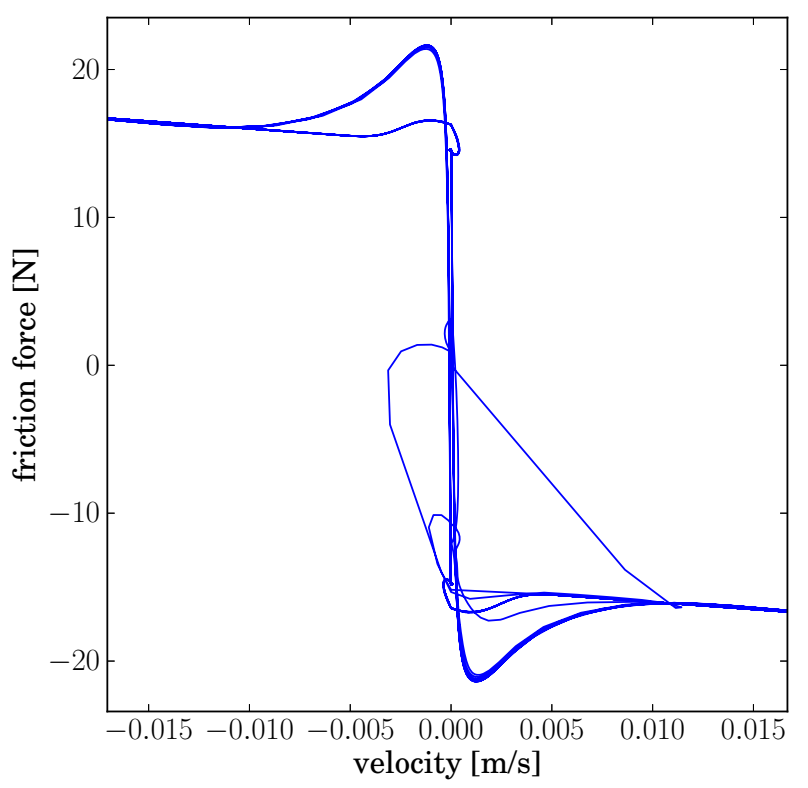

Figure 5. A trajectory (friction force w.r.t. velocity) of the Lund-Grenoble friction model

For the dynamics of the mechanism, the Modelica Multibody library as presented in Otter et al. (2003) is used. With this library, the mechanism can be represented exactly; also an extension to alternative designs can be done with little effort. Unfortunately, nonlinear systems of equations cannot be avoided at this point.

\subsubsection{Fluid domain}

For the air in the valve actuator, high-speed fluid effects can be neglected. Consequently, the Modelica fluid li- brary as presented in Casella et al. (2006) is used wherever possible.

Both valve chambers correlate to variable volume models, something not yet implemented in the Modelica fluid libary. The governing equations of a variable volume model are a generalisation of the standard volume model equations, and take the form of Equation 3, with the density $\rho$, the volume $\mathrm{V}$, and $\phi \in(1, u, \mathbf{x})$ representing mass, energy and substance balance respectively.

$$
\frac{\mathrm{d}}{\mathrm{d} t}(\phi \cdot \rho \cdot V)=\sum \text { flow }+\sum \text { source }
$$

In the case of the energy-balance, mechanical work on the cylindrical chamber volume now creates an interesting interaction between the fluid and mechanical domain. The implementation in Modelica can be seen in Listing 2.

Listing 2. Extract of Modelica code for lower variable volume model

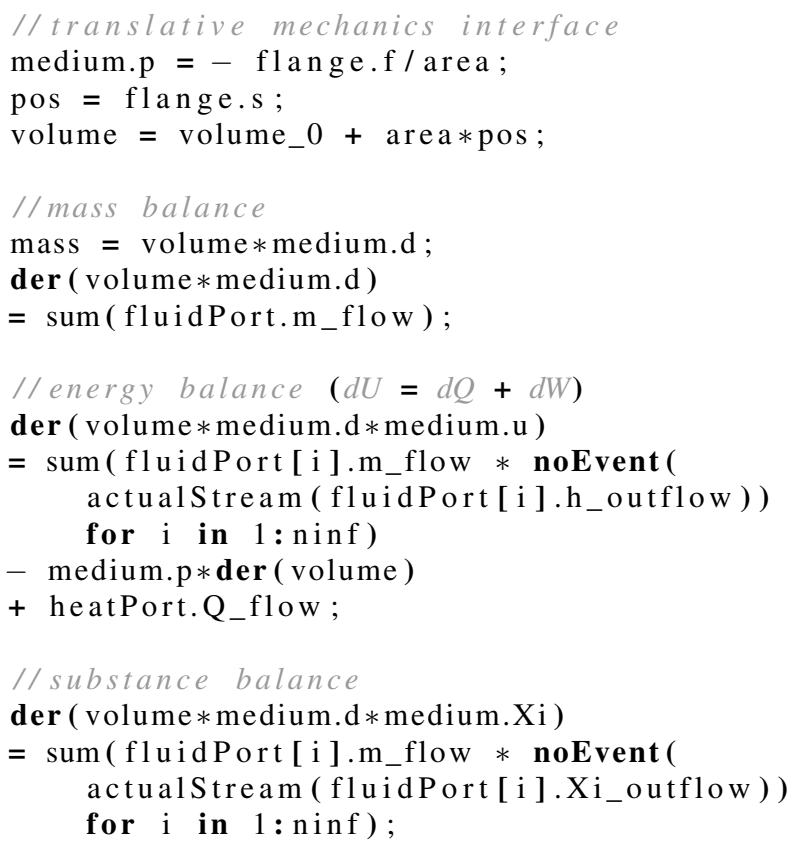

\subsubsection{Thermal domain}

The thermal effects in self-regulating pneumatic valve systems are largely dominated by the advection in the air. This is obviously already included in the fluid modelling. Nonetheless, conduction through the solid components still has to be modelled if high-fidelity results are necessary.

On the thermal side, the model is structured as follows: The environment is modelled as boundary condition of constant temperature. The actuator cylinder wall and piston are both modelled as thermal masses. A further discretization is discarded based on the high internal conductivity of the used materials. The energy dissipated by friction is added to the piston wall. Between 
the fluid volumes and the piston mass, as well as between the cylinder wall and the environment, constant thermal conductances are assumed. Between the fluid volumes and the cylinder wall, the thermal conductance is dependent on the wetted area, which is in turn dependent on the piston position.

As a consequence, a heat-conduction component was composed that connects heat conductivity with the piston position. The remainder was modelled using the Modelica thermal heat transfer library, the details of which are described by Tiller (2001).

In Figure 6, the structure of the thermal model is illustrated.

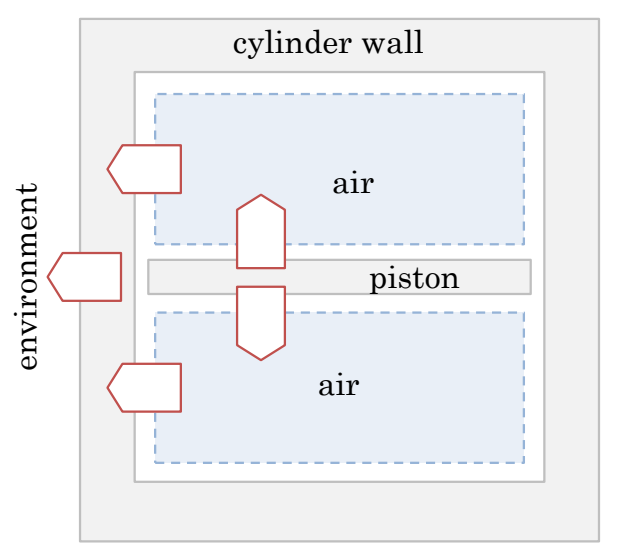

Figure 6. Thermal structure of the valve actuator

\subsection{Statistics}

The resulting models of valve and actuator feature $(0+10)$ states, $(21+161)$ time-varying variables and $(0+$ $\{3\})$ nonlinear systems of equations respectively.

\section{Results and Discussion}

\subsection{Application}

To use the model for simulations, a set of parameters has to be defined. Most of them have a geometrical meaning and can simply be taken from the specifications. For accurate results, there are however three separate measurements to be done:

\subsubsection{Friction}

In the calculation of the piston-friction as appearing in Equation 2, the Lund-Grenoble (Lu-Gre) friction model (De Wit et al., 1995) is used. In this model, the friction characteristics are defined by 6 constants. These have to be obtained from experiments or looked up in literature, based on the material-pairing.

\subsubsection{Aerodynamic Torque}

The aerodynamic torque as described in Equation 1 is dependent on the angle of the valve-disc. This dependency differs somewhat based on the geometry, but can often be estimated by CFD-calculations.

\subsubsection{Mass Flow Characteristic}

Butterfly Valves feature a S-shaped dependency between mass flow and valve angle. Like the aerodynamic torque, this dependency is only somewhat similar between valve-models. Therefore, CFD-calculations or experiments have to be deployed.

\subsection{Limit Cycle Oscillations}

For reasons of confidentiality, no actual valve setups or associated measurements can be presented here. Instead, a simpler composition is shown, where two valves are used to reduce the pressure in a pipe. The Modelica diagram of the composition can be seen in Figure 7. The pipe models are based on the gas dynamics library as presented by Sielemann (2012b). Each pipe-component represents a pipe of 20 meters length and a diameter of $0.1 \mathrm{~m}$, totalling at a length of 80 meters and a volume of around 630 liters.

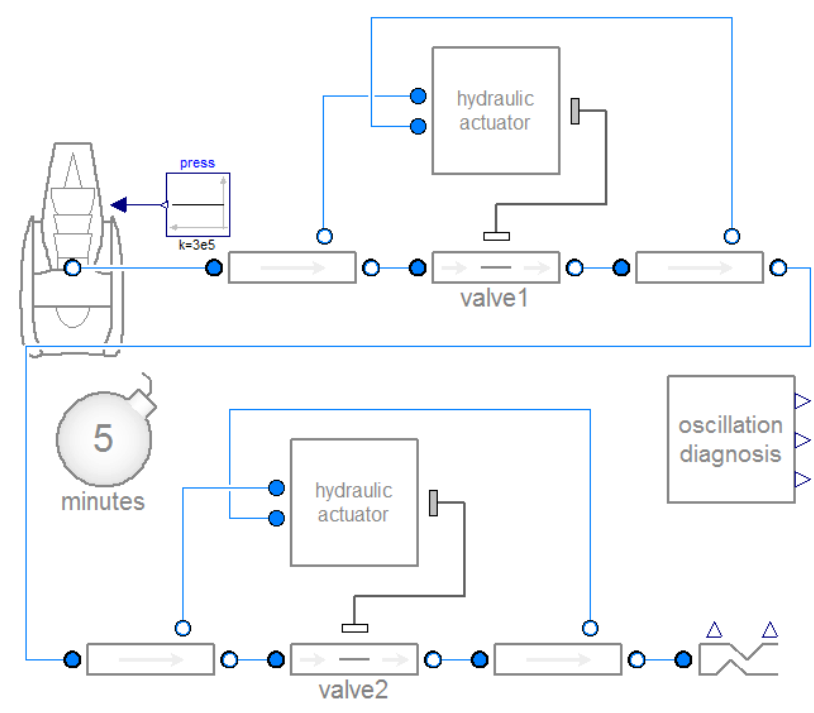

Figure 7. Modelica diagram of oscillation test case

As boundary conditions, the input pressure (left side) is set to 3 bars, while the right boundary is modelled as a quadratic resistance, normalized to a fluid velocity of $10 \frac{\mathrm{m}}{\mathrm{s}}$ at a pressure of $1 \mathrm{bar}$. The valve actuators are run in pneumatic-mode and set to regulate the downstream pressure to 2 and 1 bars respectively.

When the composite model is simulated, limit cycle oscillations occur. These are displayed in Figure 8. For both valves, the piston gets stuck at the outmost deflection, until the restoring forces are high enough to overcome the friction forces. 

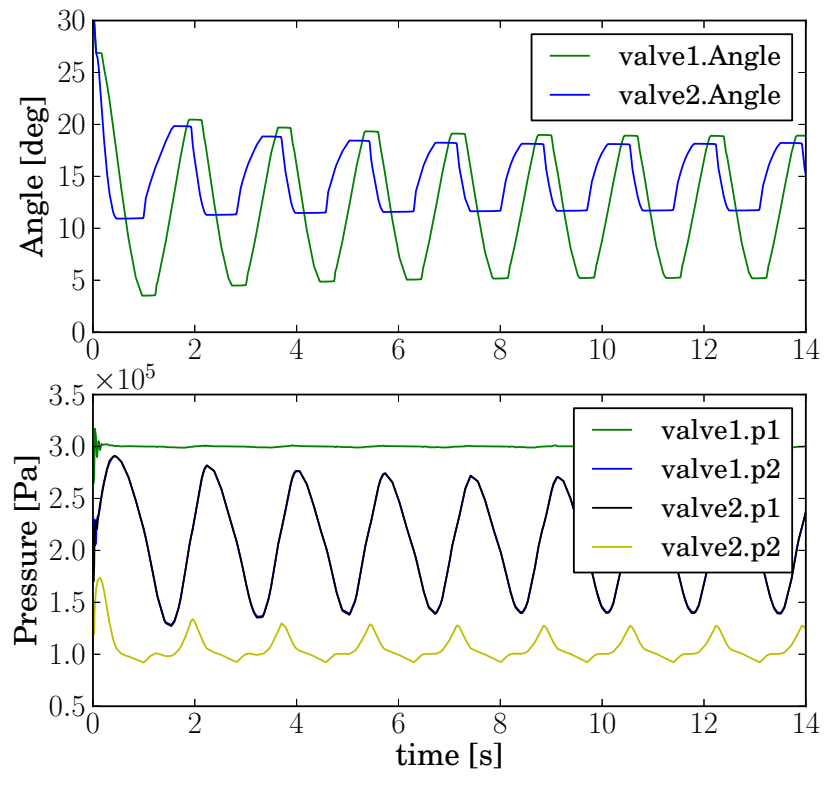

Figure 8. Results of oscillation test case

To demonstrate that the oscillations are caused by friction effects, the influence of friction and d'Alembertforces was reduced with scaling parameters. A twodimensional sweep of the quasi-steady-state amplitudes and periods over both scaling-parameters is shown in Figure 9.

It can easily be seen that the oscillations are strongly dependent on the friction forces and weakly dependent on the d'Alembert-forces. Furthermore, for vanishing friction-forces, the oscillations disappear completely. In other experiments, neglecting the d'Alembert-forces caused the oscillations to disappear, emphasising the importance of their inclusion in the model.

\subsection{Dynamic interactions}

The multi-domain nature of the presented model results in some interesting nonlinear transients. Two of them are presented in the following.

\subsubsection{Aerodynamic Torque}

The waterhammer effect is commonly known in pipeline operations. When a closing valve is used to stop the flow of a heavy and fast fluid-mass, the residual momentum of the fluid generates a build-up of pressure upstream of the valve.

For self-regulating pneumatic valves, a similar effect can occur: Let's presuppose that the valve actuator closes the valve by a particular angle. The air mass upstream of the valve is then decelerated as a result, while generating a temporary pressure build-up. This pressure-buildup in turn increases the aerodynamic torque on the valve disk, closing the disk further and amplifying the effect.
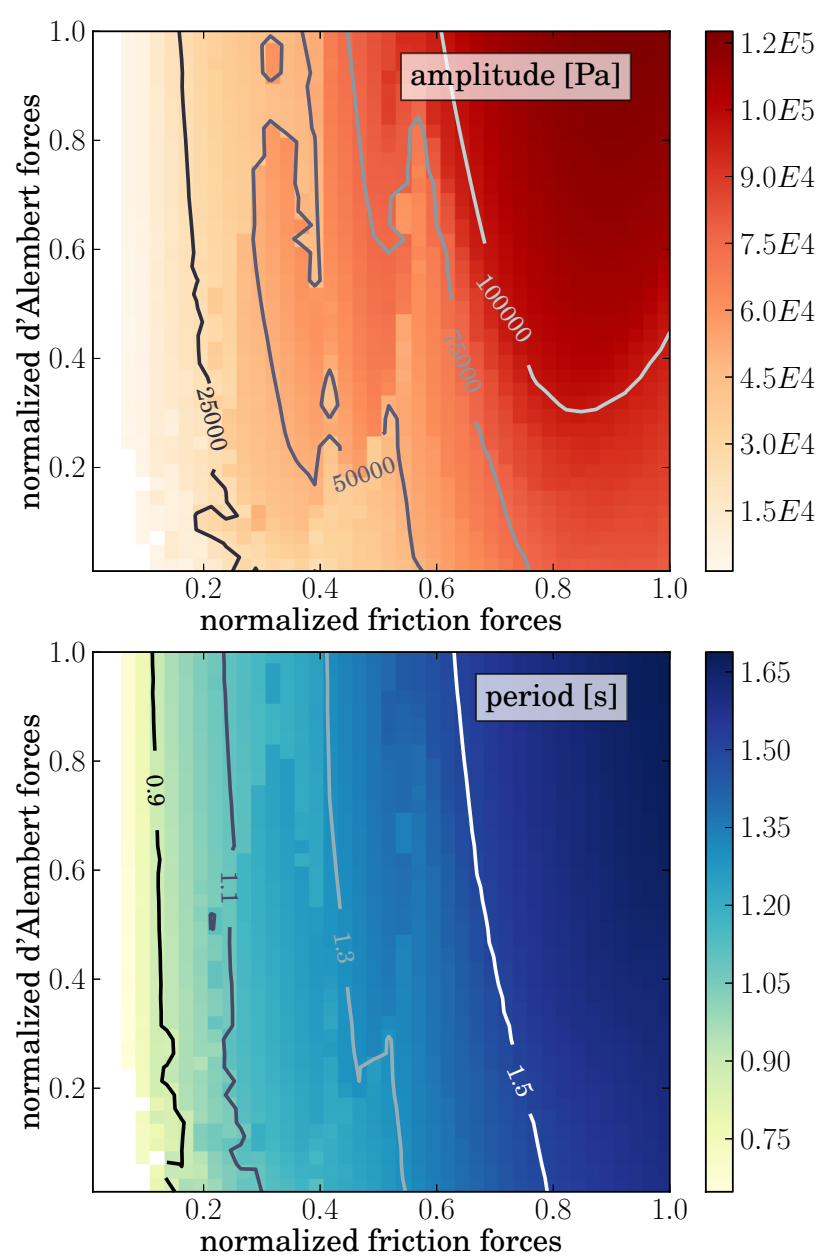

Figure 9. Results of oscillation test case

In Figure 10, a test model is represented where a pressure-regulated pipe is subjected to a harmonic inlet pressure with increasing frequency. The model was simulated with and without consideration of aerodynamic torque. The result of the simulation can be seen in Figure 11. It is easily recognizable that the valve opening is smaller when taking aerodynamic torque in consideration, especially at certain frequencies.

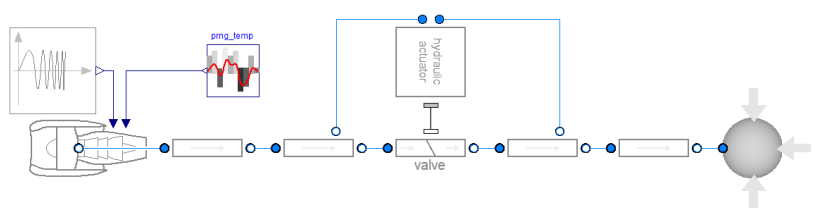

Figure 10. Aerodynamic Torque test model

\subsubsection{Oscillatory heating}

Generally, the environment of the valve has an ambient temperature different from the fluid temperature in the pipe. Also, heat conduction between environment and the valve chambers takes place. In the static case, the 

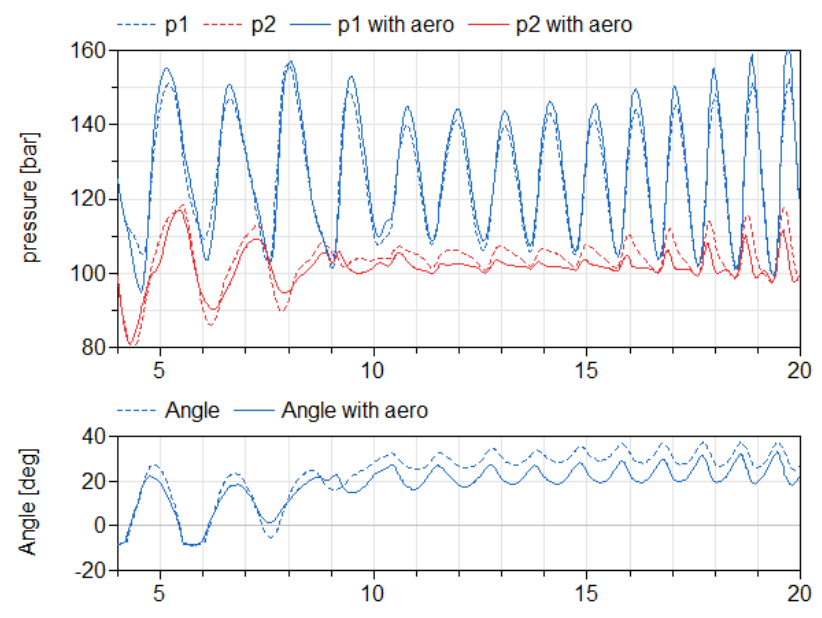

Figure 11. Transient effects of Aerodynamic Torque

temperature in the valve chamber will approach the ambient temperature after a time. However, in the case of valve movement, fluid mass is exchanged between the valve chambers and the pipe. In this way, the resulting temperature of the valve is dependent on the amount of valve movement.

\section{Conclusion}

Self-regulating pneumatic valves show a complex behavior, resulting in limit-cycle oscillations, if the overall system is not tuned satisfactorily. We present a detailed Modelica model for this kind of valves. The model includes all relevant physical effects, representing the thermal, fluid, and mechanical domains. Simulation results exhibit the typical dynamical characteristics of selfregulating pneumatic valves. Subsequently, the model can be used to predict system performance in an early development phase.

\section{References}

Martin Aberger and Martin Otter. Modeling friction in modelica with the lund-grenoble friction model. In Proceedings of the $2^{\text {nd }}$ International Modelica Conference, 2002.

Peter Beater. Modeling and digital simulation of hydraulic systems in design and engineering education using modelica and hylib. In Modelica workshop, pages 23-24, 2000.

Peter Beater and Christoph Clauß. Multidomain systems: Pneumatic, electronic and mechanical subsystems of a pneumatic drive modelled with modelica. In Paper presented at the $3^{\text {rd }}$ International Modelica Conference, 2003.

WL Bialowski. Dreams vs. reality: a view from both sides of the gap. Pulp and Paper Canada, 94:19-27, 1993.

Francesco Casella, Martin Otter, Katrin Proelss, Christoph Richter, and Hubertus Tummescheit. The modelica fluid and media library for modeling of incompressible and compressible thermo-fluid pipe networks. In Proceedings of the Modelica Conference, pages 631-640, 2006.

MAA Shoukat Choudhury, Sirish L Shah, Nina F Thornhill, and David S Shook. Automatic detection and quantification of stiction in control valves. Control Engineering Practice, 14(12):1395-1412, 2006.

C Canudas De Wit, Hans Olsson, Karl Johan Astrom, and Pablo Lischinsky. A new model for control of systems with friction. Automatic Control, IEEE Transactions on, 40(3): 419-425, 1995.

Lane Desborough and Randy Miller. Increasing customer value of industrial control performance monitoringhoneywell's experience. In AIChE symposium series, pages 169-189. New York; American Institute of Chemical Engineers; 1998, 2002.

Martin Otter, Hilding Elmqvist, and Sven Erik Mattsson. The new modelica multibody library. In Proceedings of the $3^{\text {rd }}$ International Modelica Conference. Citeseer, 2003.

Sven-Erik Pohl and Markus Gräf. Dynamic simulation of a free-piston linear alternator in modelica. In Modelica, 2005.

Aron Pujana-Arrese, Javier Arenas, Iban Retolaza, Ana Martinez-Esnaola, and Joseba Landaluze. Modelling in modelica of a pneumatic muscle: application to model an experimental set-up. In 21st European conference on modelling and simulation, ECMS, pages 4-6, 2007.

Michael Sielemann. Device-Oriented Modeling and Simulation in Aircraft Energy Systems Design. PhD thesis, Hamburg University of Technology, 2012a.

Michael Sielemann. High-speed compressible flow and gas dynamics. In Proceedings of the $9^{\text {th }}$ International Modelica Conference, 2012b.

C Solliec and F Danbon. Aerodynamic torque acting on a butterfly valve. comparison and choice of a torque coefficient. Journal of fluids engineering, 121(4):914-917, 1999.

Michael Tiller. Introduction to physical modeling with Modelica. Springer Science \& Business Media, 2001. 